August 4-7, 2013, Portland, Oregon, USA

\title{
DETC2013-13074
}

\section{PROFILING ENERGY CONSUMPTION OF SMARTPHONE USERS FOR ENVIRONMENTALLY EFFICIENT BUSINESS DECISIONS}

\author{
William Z. Bernstein ${ }^{*, \dagger}$, Devarajan Ramanujan ${ }^{\dagger}$ \\ School of Mechanical Engineering ${ }^{\dagger}$ \\ Purdue University \\ West Lafayette, Indiana, 47906 \\ Email: wbernste@purdue.edu
}

\author{
Fu Zhao ${ }^{\dagger,}$, Karthik Ramani ${ }^{\dagger, *}$ \\ School of Mechanical Engineering ${ }^{\dagger}$ \\ Wivision of Environmental and Ecological Engineering \\ $¥$ School of Electrical and Computer Engineering (by courtesy) \\ Purdue University \\ West Lafayette, Indiana 47907
}

\begin{abstract}
The recent proliferation of electronic products has led to a strong unmet need for understanding environmental implications of new technologies. According to Nielsen, 66\% of Americans ages 24-35 own a smartphone, providing strong evidence that this technology can be considered ubiquitous. However, a majority of users and service providers are not sensitive to energy implications of data usage. As parameters affecting data delivery and usage are primarily driven by user behavior, this study is focused on using empirical data to investigate its correspondence to energy footprint. A large cohort of smartphone users $(n=21,853)$ and the means for which data is accessed, i.e. via $3 G / L T E$ or WiFi, is examined to develop a model for estimating the energy cost of the various modes of data usage. Finally, alternate business scenarios are developed through simulating behavior change in cohorts based on existing data and then assessed with regards to economic and environmental efficiencies.
\end{abstract}

\section{INTRODUCTION}

Over the last decade, the world has seen an unprecedented proliferation of electronic products. From tablet computing devices to televisions, electronics have become easily and readily available to a great number of people around the world. Mobile phones, in particular, have strongly exhibited this trend. Accord-

\footnotetext{
*Address all correspondence to this author.
}

ing to the International Telecommunication Union (ITU), there were six billion mobile subscriptions at the end of 2011. Interestingly, both China and India, two rapidly growing economies, each accounted for about one billion subscriptions [1]. When also considering $3 \mathrm{G}$ penetrance, or third generation mobile telecommunications technology, the numbers remain significant. Currently, it is estimated that $3 \mathrm{G}$ technologies account for an estimated $15 \%$ of all cellular subscriptions worldwide, including $70 \%$ in some countries, e.g. Japan (99\%), Spain (85\%), Italy $(83 \%)$ and the United States (81\%) [2]. All in all, the ITU estimates that there were 1.1 billion mobile Web users in the world in 2011. On-demand data via the Web is no longer a commodity or a privilege in many societies but instead it is seen as a right for all people to be able to access. As a result, smartphones, which can be defined as "cellular telephones with built-in applications and Internet access" [3], have seen a steep increase in sales due to strong user demand. According to Nielsen, 66\% of Americans ages 24-35 own a smartphone [4], and smartphone penetrance is expected to rise.

In turn, the energy consumption of the information communications and technology (ICT) industry has seen a significant rise due to the ubiquity of mobile devices as described above. For example, in the United States alone, the energy consumption of the network equipment was estimated to have used between 14 and $18 \mathrm{TWh}$ in 2008 and is expected have grown to $23 \mathrm{TWh}$ in 2012, assuming business-as-usual [5]. Furthermore, the green- 
house gas emissions due to the ICT industry is expected to grow from $300 \mathrm{Mt} \mathrm{CO} 2$ in 2007 in $350 \mathrm{Mt} \mathrm{CO} 2$ emitted in 2020 [6]. However, it seems that a majority of users and service providers are not sensitive to the energy-related implications of data usage.

In general terms, the trends of smartphone user behavior have recently been investigated. Contrary to mobile phone users in the past, i.e. $2 \mathrm{G}$ subscribers, the extended capabilities of advanced platforms, e.g. on-board GPS and on-demand e-mail browsing, have shifted user behavior. There seems to be a trend that more time is being spent running apps on smartphones than checking mail, placing calls, or any other activity [7]. In general, it has been suggested that user behavior is a hurdle for sustainable development. However, there has been little work related to emerging business models that link user behavior and energy consumption through various types of ICT infrastructure. For example, if one suggests that electronic publishing of an article is beneficial for the environment, the publishers still cannot control users from both downloading and printing that article for personal use [8].

This paper focuses on examining empirical data in order to better understand user behavior and suggest alternative business scenarios from the service provider perspective aiming to curb energy consumption during the product's use. The paper presents a case study of smartphones in which empirical data for monthly use of over 20,000 smartphone subscribers $(n=21,853)$ is analyzed. As parameters affecting data delivery and usage are primarily driven by user behavior, this study is focused on using empirical data to investigate its correspondence to energy footprint. Alternate business scenarios are developed through simulating behavior change in cohorts based on existing data and then assessed with regards to economic and environmental efficiencies. The paper is closed with remarks that consider environmental implications of cyber-physical products as a whole.

The data used throughout this manuscript was provided by Mobidia Technologies, Inc, a venture-funded corporation, that has worked with notable ICT-related companies, e.g. Microsoft, Symbian, Motorola, Samsung, LG and PMC-Sierra [9]. Mobidia offers a free app, titled "My data manager", which allows smartphone users to essentially track their data usage, i.e. WiFi or $3 \mathrm{G} / 4 \mathrm{G}$ access, in order to avoid additional costs related to overages. A summary of data collected can be found in [10], published as a white paper for the public.

\section{RELATED WORK}

The explosion of smartphone use has spurred work related to user behavior. A recent periodical in the Harvard Business Review listed the seven primary motivations for smartphone use as self-expression, discovery, preparation, accomplishing, shopping, socializing, and "me time" [11]. One significant finding of the HBR study is that $68 \%$ of consumers' smartphone use occurs at their home, suggesting that a significant amount of the data ac- cessed could be via a home WiFi network. One group focused on developing an on-board app that keeps track of data usage with regards to many categories [12], similar to the software developed by Mobidia. Kivi, 2007 also studied the measurement of mobile user behavior and service usage through surveys [13].

Similar to this manuscript, some of the related research are targeted towards understanding large-scale smartphone use. Oliver, 2010 conducted a study with a large cohort of BlackBerry users $(n=17300)$ and measured the duration of time spent by users [14]. Falaki et al. investigated user behavior and its effect on phone energy consumption in order to develop predictive models that can better help increase battery life [15]. Another study took a close look at the energy consumption of specific hardware components, e.g. GSM, CPU, RAM, WiFi, etc, during different power states of the phone in order to make redesign suggestions of individual hardware components [16].

Modifying user behavior, in general, has been an area of research and study for quite some time. Recently, work has been focused on changing user behavior through design exploiting human natural behaviors [17]. In the context of web search, researchers have shown interest into understanding keywords or entry behavior to quickly retrieve requested information [18]. A similar objective has been used for developing rules for stronger passwords for sensitive information [19]. Shiraishi et al. developed a application that visualize energy costs and emissions of their home via virtual avatars to curb user behavior [20]. One study reviewed methods used to change user behavior with respect to responsible library treatment [21]. Other studies have investigated changing user behavior to enhance traffic safety $[22,23]$

It should be noted that not one of the before-mentioned studies investigated the effect of modifying user behavior in order to curb the IT industry's energy consumption. However, there have been other studies to have mentioned smartphone user behavior in this context. Radia et al. binned users based on their monthly limit of allowable cellular data in order to understand traffic of particular types, e.g. file sharing, on-line media, and web browsing, and to formulate suggestions related to developing apps to make users more contextually aware of their usage [24]. Heinemann et al. grouped users into two behavioral categories, i.e. users who share everything and ones that share nothing, in order to suggest data loading schema for dissemination schemes [25]. Others have investigated smartphone charging behaviors to understand typical user activity with respect to the energy consumed by hardware components and subsequent network connectivity characteristics [26]. Another study took more of a marketing approach and measured a typical user's daily time allocation on various mobile services, application installations, and communication actions. Interestingly, the study found that $44 \%$ of those surveyed actively follow cellular data rates and modify behavior accordingly [27].

To the best of the authors' knowledge, there have not been 
TABLE 1. NUMBER OF REPORTED USERS PER DATA PLAN BUCKET

\begin{tabular}{|c|c|}
\hline Data Plan (GB) & Population (n) \\
\hline $\begin{array}{ll}0.0 & 0.3\end{array}$ & 2131 \\
\hline 0.30 .5 & 43 \\
\hline 0.51 .0 & 175 \\
\hline 1.03 .0 & 5757 \\
\hline 3.05 .0 & 2846 \\
\hline Over 5.0 & 226 \\
\hline Unlimited & 1766 \\
\hline Did not respond & 8909 \\
\hline
\end{tabular}

studies yet that present schema for assessing feasible modifications to user behavior through new business models in order to make users more sensitive to the energy consumption of data acquisition. This study, aims to create such scenarios by investigating a large set of empirical data.

\section{SMARTPHONE USER DATA OBSERVATIONS}

The provided data from Mobidia was organized primarily based on the purchased data plan, since it is expected that the ceiling of cellular data usage per user would have the most significant contribution to user behavior in the context of accessing data. To understand the general trends of smartphone usage, each user was binned in groups based on their self-reported data plans. The seven bins for the data plans can be seen below in Table 1 . The group with the most amount of users reported a data plan limit of somewhere in the range of 1.0 and 3.0. Most of these users have a 2.0 GB limit, as this is a common data plan offered throughout the service provider industry. It should be noted that many users refrained from providing their data plan information $(\mathrm{n}=8909)$. This sub-population was not included in any analysis throughout the paper. In general, the dataset was considered acceptably representative of an aggregate user population.

The Mobidia app used in this study provides various characteristics of each user, such as the user's operating system (OS) type, phone manufacturer, phone model, service provider, aggregate monthly data usage via $\mathrm{WiFi}$, and aggregate monthly data usage via $3 \mathrm{G}$ or LTE (i.e. $4 \mathrm{G}$ ) depending on the phone model. It was assumed that all users are representative of a population of $3 \mathrm{G}$ operating users, i.e. users with LTE access were grouped together with smartphone operators using $3 \mathrm{G}$ to access cellular data. It should also be noted that the only data used from the monthly data set is the aggregate cellular and WiFi usage. Re-

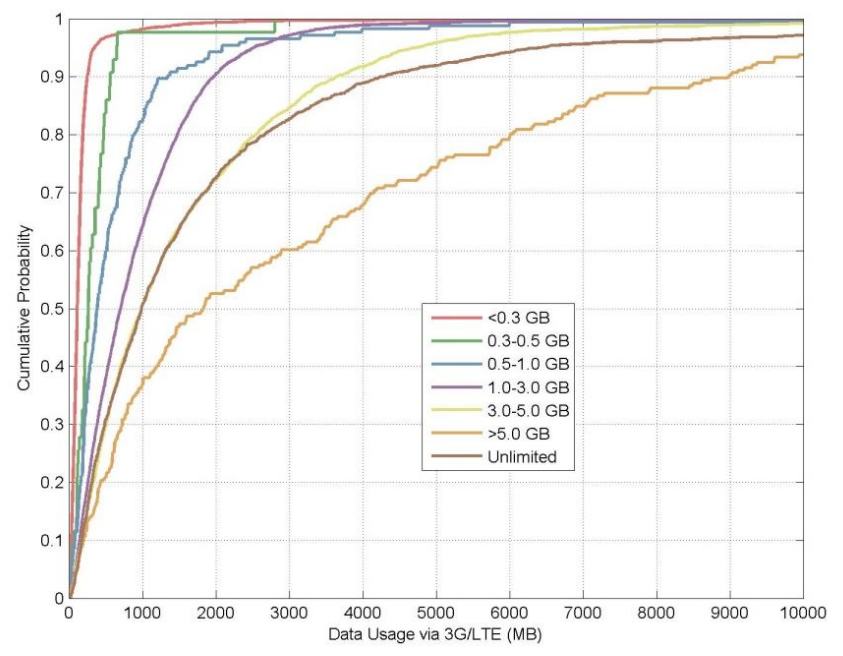

FIGURE 1. CUMULATIVE PROBABILITY DISTRIBUTIONS FOR DATA ACCESSED VIA THE CELLULAR NETWORK

gardless of the type of smartphone model, OS type, carrier, etc., each user was considered to be representative of a large cohort of $3 \mathrm{G}$ users in order to make observations of the studied populations.

Figures 1 and 2 organize raw data with respect to data acquisition via $3 \mathrm{G}$ across the studied data plan buckets. Figure 1 represents a plot of the cumulative distribution functions (CDF) of each data plan cohort, excluding the users that abstained from providing their data plans. Figure 2 shows triangular kernel smoothing density estimates for each bucket as in Figure 1. The

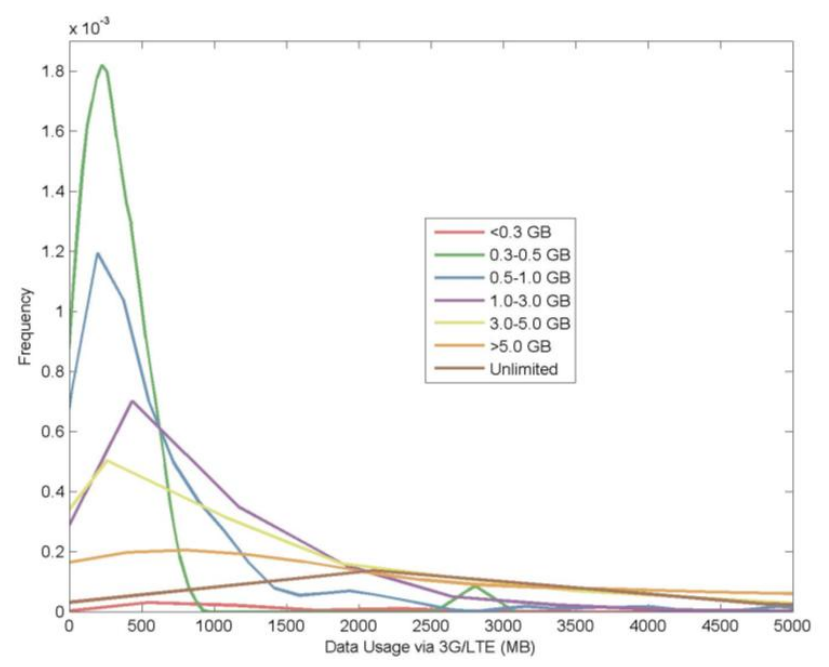

FIGURE 2. TRIANGULAR KERNEL SMOOTHING DENSITY ESTIMATES FOR DATA ACCESSED VIA THE CELLULAR NETWORK 


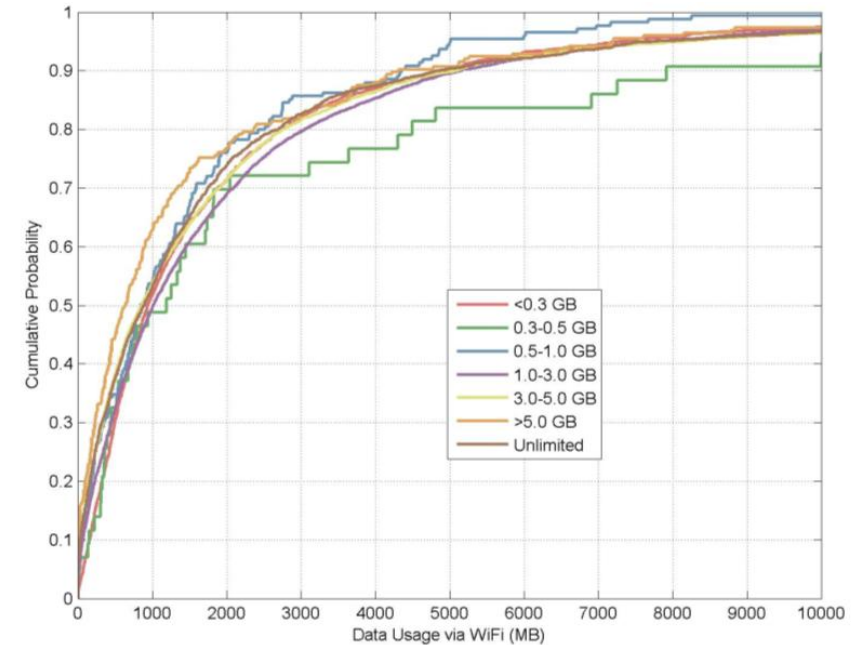

FIGURE 3. CUMULATIVE PROBABILITY DISTRIBUTIONS FOR DATA ACCESSED VIA WIFI

main purpose of observing the CDF is to understand the relative fraction of data usage per user group. However, the density estimates provide a visualization scheme for understanding frequency of data usage. For instance, a tight peak will represent a large subset of users behaving in a particular manner. More specifically, the triangular kernel smoothing method (i.e. the ksdensity function [28]) available in Matlab's Statistics Toolbox is used to represent corresponding histograms of each dataset [29]. For ease of comparison, the color scheme for each respective bucket shown in Figures 1 and 2 will be the same throughout this manuscript. Similarly, Figures 3 and 4 show the cumulative

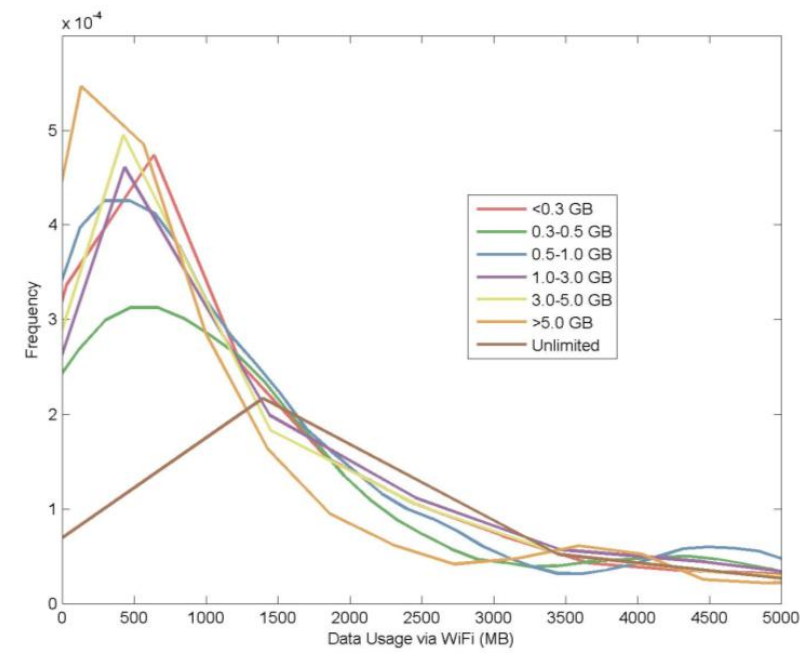

FIGURE 4. TRIANGULAR KERNEL SMOOTHING DENSITY ESTIMATES FOR DATA ACCESSED VIA WIFI probability distributions and triangular kernel smoothing density estimates, respectively, of the monthly Wifi usage for each studied data bucket.

As seen in Figures 1 and 2, data accessed via smartphones from $3 \mathrm{G}$ varies across data plans. This can be particularly evident when comparing significantly different data plans, e.g. less than $300 \mathrm{MB}$ and over $5 \mathrm{~GB}$. From the CDF of the $3 \mathrm{G}$ usage in Figure 1 , it can be seen that almost $50 \%$ of users with plans over $5 \mathrm{~GB}$ access at least $2 \mathrm{~GB}$ of data via $3 \mathrm{G}$ monthly as opposed to some $1 \%$ of those with plans less than $300 \mathrm{MB}$. This should not be a surprise considering that users with low limit plans are much more sensitive to costs related to exceeding data limit ceilings. Observing Figure 2, it is apparent that those with smaller plans, e.g. between than $300-500 \mathrm{MB}$, exhibit similar behavior shown as a tighter peak. As the data plan increases, the smoothing estimates seem to level out, suggesting that users with high-range plans exhibit behavior more difficult to predict.

On the contrary, with respect to data obtained via $\mathrm{WiFi}$, shown in Figures 3 and 4, it seems that WiFi usage is quite similar irregardless of data plan size. From Figure 3, one might think that the group of users that have plans that fall within the range of 300 and $500 \mathrm{MB}$ show unique behavior in terms of WiFi usage, but this seems to be due to the low sample size, as there are only 43 such users, compared to 5757 users that have a 1-3 GB plan, for example. If this is not an artifact of low sample size, one explanation could be that those with lower cellular data plans do not necessarily access lower amount of data than others, but procure a higher percentage of their traffic through WiFi rather than $3 \mathrm{G}$. These users, hence, may exhibit higher sensitivity to cost overages, enforced once they exceed their allowable data ceiling. One can also see that the percentage of users that exceed

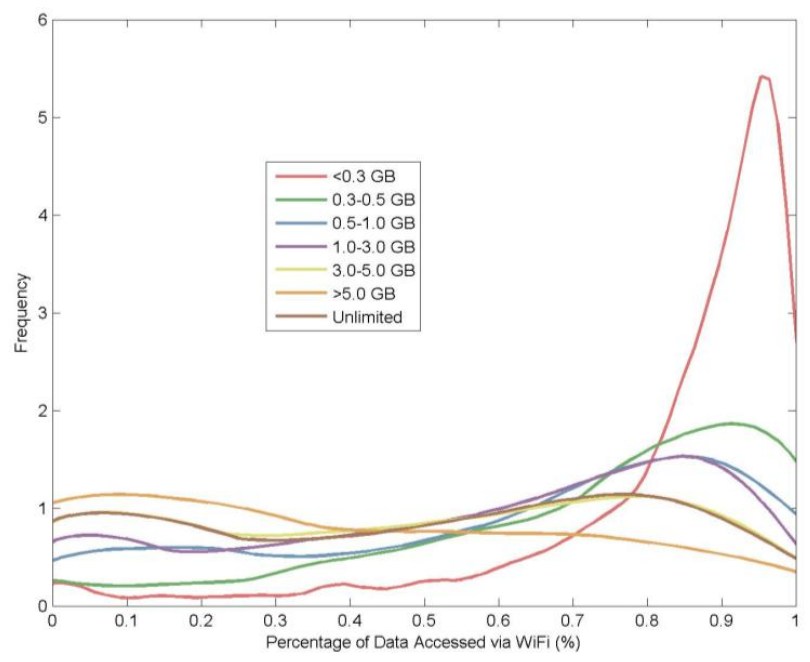

FIGURE 5. TRIANGULAR KERNEL SMOOTHING DENSITY ESTIMATES OF PERCENTAGE OF DATA ACCESSED VIA WIFI 
their data plan for higher data plans, e.g. in the 3-5 GB bucket, compared with lower ones, e.g. 0.3-0.5 GB bucket, seems to be slightly higher. Figure 5 illustrates this idea in more detail by showing trends of WiFi to $3 \mathrm{G}$ usage among groups. Those with lower plans are much more sensitive in keeping their ratio of WiFi data usage to $3 \mathrm{G}$ data usage high, while there is little distinction among users with larger data plans. Other distinctions between user groups and general observations of the data can be seen in the white paper sponsored by Mobidia [10].

\section{DATA LIMITATIONS}

It should be noted that the empirical data used throughout this manuscript is not without limitations. First off, those who download the app from Mobidia can be categorized as high-level users, simply by the fact that they must possess enough expertise to download and use an energy-monitoring app. This is not reflective of all smartphone users, as many novice users may have trouble downloading such apps or lack motivation. Furthermore, these smartphone users could develop more sensitivity to their own data use once they have deployed Mobidia's app on their device. In other words, being provided on-demand access to their data usage could modify their behavior. Another limitation is the large set of users who abstain from reporting their data plan, cell phone and other characteristics that have been used to analyze the data. Of the 21,853 users that were analyzed, 8909 of them refrained from reporting any information to Mobidia. Additional analysis, however, illustrates that the abstaining users closely fit behavior of the others analyzed in total, suggesting that these users could be considered good representatives of a population of smartphone users with mixed data plans. In other words, the cumulative density functions and histograms match closely between the abstaining users and the rest of the studied population. Also, all data collected is reflective of usage within the month of May 2012. It is not clear, however, whether the data fluctuation from month to month is significant.

\section{MODELING USE PHASE ENERGY CONSUMPTION}

To the authors' knowledge, the most rigorous life cycle assessment, to date, of the $3 \mathrm{G}$ network infrastructure was conducted by [30]. Within the study, the authors argued that as $3 \mathrm{G}$ mobile phones become ubiquitous, similar to the current situation, the impact per bit related to network components decreases significantly. At the time of the study, the analysis was conducted assuming $3 \mathrm{G}$ subscribers were in the range of 1-4 million. As of Q4 in 2011, there are 208 million 3G subscribers in the US alone [31]. This suggests that the manufacturing and operation of mobile phones themselves is becoming more critical with respect to the environmental efficiency of the telecommunications industry as a whole. Hence, here the focus will remain on the operation of the devices and the energy they consume while access-
TABLE 2. ENERGY MODEL FOR DOWNLOADING X KB OF DATA OVER 3G AND WiFi ON A SMARTPHONE, ADOPTED FROM [2]

\begin{tabular}{ccc}
\hline & $3 \mathrm{G}$ & WiFi \\
\hline Transfer Energy $(\mathrm{J})$ & $0.025(\mathrm{x})+3.5$ & $0.007(\mathrm{x})+5.9$ \\
Tail Energy $(\mathrm{J} / \mathrm{s})$ & 0.62 & N/A \\
Maintenance $(\mathrm{J} / \mathrm{s})$ & 0.02 & 0.05 \\
Tail Time $(\mathrm{s})$ & 12.5 & N/A \\
\hline
\end{tabular}

ing data. Furthermore, estimating energy consumption through the network infrastructure is infeasible without accessibility to the infrastructure components themselves. For this reason, much of the work in regards to estimating energy per bit transfered is focused on the end user device, i.e. the smartphone itself.

A widely accepted publication in particular, [2], provides a linear energy model based on empirical measurements of a smartphone. The model presented in [2] is illustrated in Table 2. The transfer energy shown in the table estimates the amount of energy required to "ramp up" to the required power level as well as the actual energy expended during transmitting and receiving the data. For WiFi, the transfer energy includes both transmitting data as well as the energy required for scanning and association. The tail energy estimation describes the expenditure of energy as the device lingers at high power states. This value should not be ignored since it contributes to some $60 \%$ of the total energy with respect to a single $50 \mathrm{~K}$ download. The linear energy model here was used to model the energy consumption of each $3 \mathrm{G}$-operated smartphone. As seen in the Table 2, the model requires length in time for each download with specific packet sizes. From the data used in this study, these numbers cannot be found and estimating packet size could introduce additional uncertainty. Therefore, a factor of 2.2 (i.e. $100 \% / 40 \%$ ) applied to the transfer energy is used to recover a reasonable estimate. If this factor were not included, the energy consumption of $3 \mathrm{G}$ access would be significantly underestimated.

The cumulative distribution functions and the triangular kernel smoothing density estimates representing the energy consumption per user are shown in Figures 6 and 7. These plots were created to observe apparent trends. It is interesting to note that, in terms of energy consumption, that the four lowest data plans, (1) less than $300 \mathrm{MB}$, (2) between 300 and $500 \mathrm{MB}$, (3) between 0.5 and 1.0 GB and (4) between 1.0 and 3.0 GB, exhibit similar behavior as seen in Fig. 7. The other plans have significant tails to the right, which represents users with heavy usage needs.

The model can be validated by observing holistic consumption. According to the energy model, the estimated total monthly end-user energy consumption of the studied users $(n=12944)$ is 


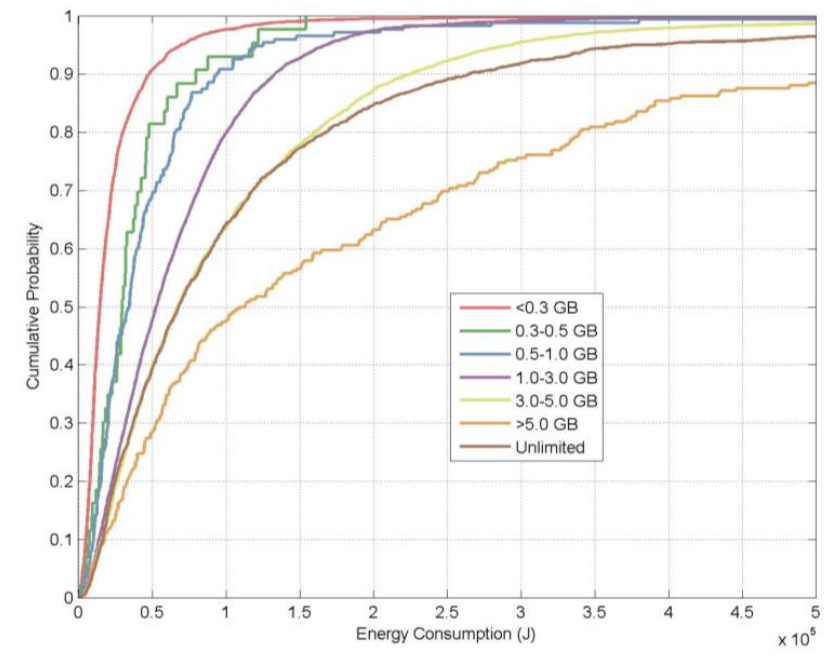

FIGURE 6. CUMULATIVE PROBABILITY DISTRIBUTIONS OF ESTIMATED ENERGY CONSUMPTION

about $1.05 \mathrm{TJ}$, which averages to about $81.1 \mathrm{KJ}$ monthly per user, or $270 \mathrm{Wh}$ yearly. Using the electricity rate of about $\$ 0.093$ per $\mathrm{kWh}$ [32], the energy consumption related to accessing data is on the order of cents under given assumptions, i.e. \$0.025. This compares with other estimates reported elsewhere, as in [33].

It should be noted that although it is not done here, it is possible to extend the energy model across the network components of both a $3 \mathrm{G}$ and WiFi setup. Although without direct measurement of components which dominate life cycle consumption, i.e. the antenna stations, and the antenna station controllers [30,34], gross assumptions must be made. One could use an efficiency index, called power usage effectiveness (PUE) defined as the total energy consumption divided by the IT equipment energy consumption and obtain a gross estimate of per-user energy consumption across the $3 \mathrm{G}$ network, as in [35].

\section{BUSINESS SCENARIOS FOR MITIGATING USE-PHASE ENERGY CONSUMPTION}

In order to institute business strategies in this context, it is necessary to understand the future of the industry. Smartphone penetration in the US is expected to continue to rise. As a result, there is strong evidence that service providers will not be able to match infrastructure constraints to meet this need [36]. This trend is evident as service providers are expanding their WiFi Hotspots availability [37]. As a result, it is assumed that it could be economically viable to force users on to WiFi through additional cost penalties in order to avoid new infrastructure cost. This presents a counterintuitive situation, since overage costs are profitable for service providers. However, less base station towers to cover more subscriptions is economically beneficial, creat-

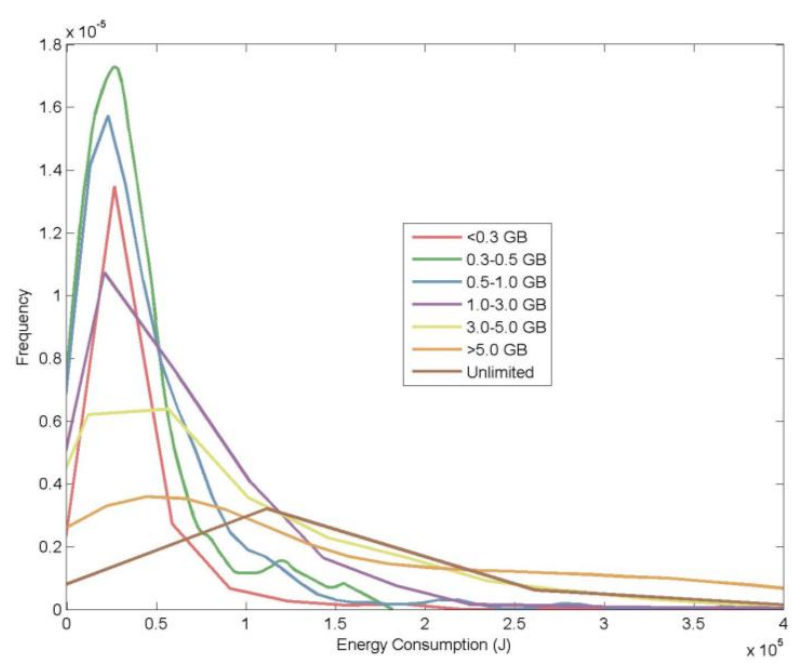

FIGURE 7. TRIANGULAR KERNEL SMOOTHING DENSITY ESTIMATES OF ESTIMATED ENERGY CONSUMPTION

ing an increase in value per user to the provider.

From the energy model in the previous section, it is clear that data accessed via WiFi exhibits lower end-user energy consumption profiles than via $3 \mathrm{G}$. One can generalize four main user groups defined by their user behavior, users that (1) use low amounts of $3 \mathrm{G}$ data as they approach their limit due to fear of overage costs, (2) use heavy amounts of data while exhibiting limited overage cost sensitivity, (3) use low amounts of data even though their plan allows higher data usage rates, and (4) use heavy amounts of data closely fitting to their plan. The grouping of these user groups can be seen in Fig. 8. These groups are

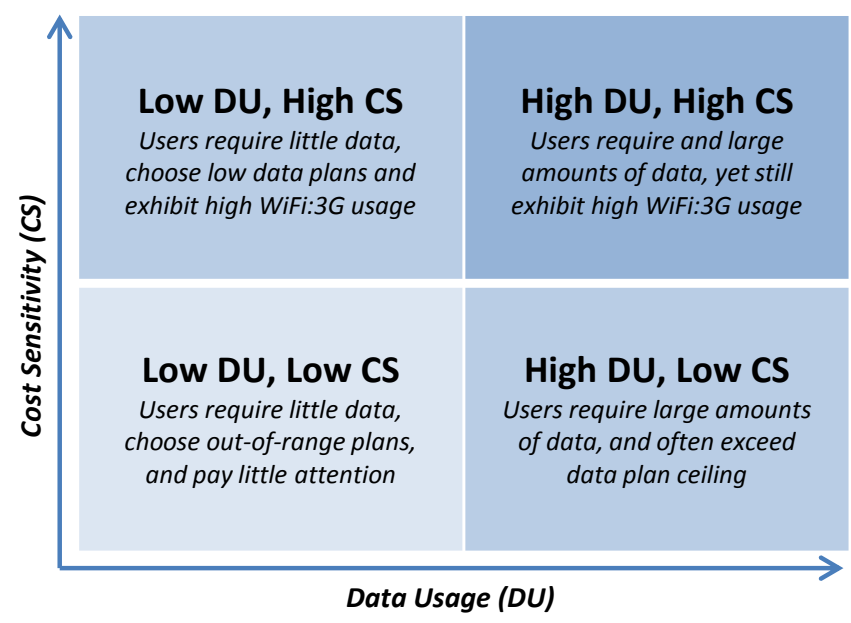

FIGURE 8. QUADRANT CHART OF USER BEHAVIOR 


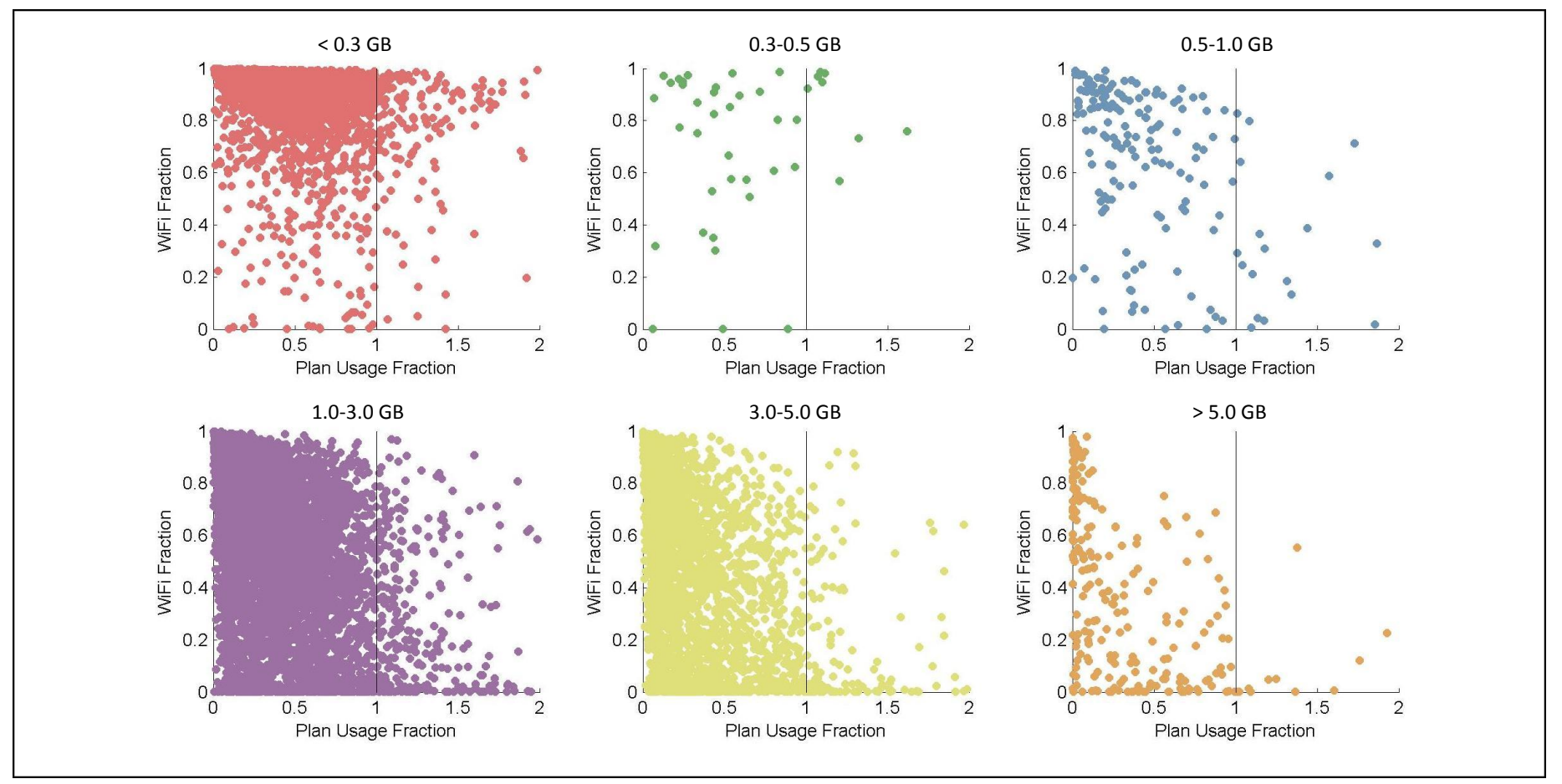

FIGURE 9. PLOTS OF THE PLAN USAGE FRACTION (PUF) AGAINST THE WIFI FRACTION (WF), WHICH CAN CHARACTERIZE BEHAVIOR PROFILES FOR SELECT DATA PLAN BUCKETS

formed by assessing their general trends over two criteria, their data usage requirements (shown on the $\mathrm{x}$-axis) and their cost sensitivity (shown on the y-axis). Cost sensitivity can be defined as the degree for which users respond to overage costs. In other words, those very high cost sensitivity will theoretically never exceed the allotted $3 \mathrm{G}$ data plan. On the other hand, one can see trends of users exhibiting low cost sensitivity, often exceeding their alloted 3G data. Data usage here is defined by the sum of data accessed via WiFi and 3G. In this specific application, abstraction is useful since usage patterns can now be categorized and simplified in meta-groups.

Here, behavior profiles for each data plan bucket are presented in order to match these meta-groups. Users with unlimited plans are excluded since they will exhibit obvious behavior that is insensitive to data usage. Each profile is created by calculating two key attributes of each user, (1) the plan usage fraction (PUF) and (2) the WiFi fraction (WF).

$$
\begin{gathered}
P U F=\frac{D_{3 G}}{D_{3 G, c e i l}} \\
W F=\frac{D_{W i F i}}{D_{3 G}+D_{W i F i}}
\end{gathered}
$$

where $D_{3 G}$ is the amount of monthly $3 \mathrm{G}$ data, $D_{3 G \text {,ceil }}$ is ceiling of the specific user's data plan, and $D_{W i F i}$ is the amount of monthly WiFi data usage.

Fig. 9 illustrates the behavior profiles, defined as PUFvs.WF for all users. The users are plotted separately according to data plan in order to show relative trends of behavior. Each plot also includes a vertical line at the value of $P U F$ equal to 1 , which represents the point at which a user exceeds their data plan and experiences overage charges. One can see that there is a significant percentage of users who exceed their data plans, i.e. $12.3 \%, 20.9 \%, 16.6 \%, 5.5 \%, 4.8 \%$ and $4.8 \%$ for data plans of lesser than 0.3 GB, 0.3-0.5 GB, 0.5-1.0 GB, 1.0-3.0 GB, 3.0-5.0 GB and those greater than 5.0 GB, respectively. Interestingly, those that exceed their data plans seem to exhibit unique behavior with respect to which data plan they belong. For the data plans lesser than $300 \mathrm{MB}$, we see a cluster of users that exceed their data plan, i.e. beyond $P U F$ equal to 1 , yet still have a heavy WiFi:3G ratio. These users can be seen in the upper-right quadrant of their respective plot in Fig. 9. This is quite different compared with users that hold data plans greater than 1.0 GB. In these plots, there is a cluster of users, who again exceed their data plan ceiling, yet have very low WiFi:3G ratios. This can be contributed to two possible reasons, (1) these users have limited access to WiFi throughout their daily activities or (2) they simply 
TABLE 3. SUMMARY OF POTENTIAL ENERGY SAVINGS FOR ALTERNATE BUSINESS SCENARIOS

\begin{tabular}{cccc}
\hline Business Scenario & Potential Savings of Studied Users & Percent of Total Consumption & Potential Savings Over Entire US \\
\hline 1 & $16.5 \mathrm{MJ}$ & $1.6 \%$ & $0.86 \mathrm{GWh}$ \\
2 & $22.5 \mathrm{MJ}$ & $2.1 \%$ & $1.13 \mathrm{GWh}$ \\
3 & $46.4 \mathrm{MJ}$ & $4.4 \%$ & $2.39 \mathrm{GWh}$ \\
\hline
\end{tabular}

are not sensitive to overage costs. From a business perspective, the potential of energy savings lies within users in large plans that exceed data plans, yet exhibit a moderate WiFi:3G ratio. It should be noted that an appropriate WiFi:3G ratio indicator for users who have limited WiFi access is not very clear. Nevertheless, the following three business scenario projections aim to take advantage of these user types.

Business Scenario 1 investigates the potential of changing behavior of those with a moderate $\mathrm{WiFi}: 3 \mathrm{G}$ ratio, defined as $0.1<W F<0.9$. Assuming that service providers institute cost penalties for exceeding their data plan, users would became more sensitive to overages. In this case, it is assumed that existing $3 \mathrm{G}$ overages be converted to $\mathrm{WiFi}$ data access. The energy model was simulated by swapping overage $3 \mathrm{G}$ usage with WiFi and potential energy savings were calculated to be about $16.5 \mathrm{MJ}$, or $1.6 \%$ of the total energy consumption.

Business Scenario 2 presents a similar situation as the previous scenario. Here, additional users on the WiFi:3G spectrum, i.e. $0.05<W F<0.95$, are included. The potential savings in this scenario were estimated to be $22.5 \mathrm{MJ}$, or $2.1 \%$ of the total energy consumption. Again, this could be achieved with more significant cost penalties.

Business Scenario 3 investigates opportunity of modifying behavior of the users with unlimited data plans and instituting the rules of Business Scenario 2. It is assumed that those with unlimited plans change to data plans of $3 \mathrm{~GB}$, and exhibit high sensitivity to overages. Here, a more significant savings is recorded, i.e. $46.4 \mathrm{MJ}$ or $4.4 \%$ savings.

In total, if smartphone users across the US fit within these characteristics of the data presented in this paper, a significant amount of potential energy savings exist. Assuming 200 million $3 \mathrm{G}$ smartphone users, a rate of $270 \mathrm{Wh}$ per year, a low percent savings estimate of $1.6 \%$ and a high percent savings estimate of $4.4 \%$, a potential energy savings exists of between $0.86 \mathrm{GWh}$ and $2.39 \mathrm{GWh}$ yearly, just from end-user energy consumption on the device itself. In order to understand the scale, $1 \mathrm{GWh}$ can meet the yearly energy demand of about 100 US homes. These results are summarized in Table 3.

The numbers presented in this section dictate that even without shifting actual user behavior in terms of the amount of data accessed, there is significant potential savings in providing addi- tional WiFi access to users. Of course, in order to understand the business implications of these changes, a full cost analysis necessary. All relevant factors such as total monthly cost to the user, including basic and overage costs, would have to be included to validate that instituting cost penalties as viable strategies.

\section{TOWARDS UNDERSTANDING ENVIRONMENTAL FOOTPRINTS OF CYBER-PHYSICAL SYSTEMS}

This paper presents a scenario for which user behavior plays a key role in determining a product system's energy footprint. The smartphone example can be related to a more general group of products, namely smart products. A smart product can be defined as a networked physical (or cyber-physical) device that has means of acquiring, processing and delivering contextualized information in order to enable behavior modification of itself or its user. In this sense, smart products can be viewed as a subcategory of cyber-physical systems (CPS), as CPS use computational, often distributed resources in order to enhance some physical entity. In general, environmental or energy analyses of CPS have been scarce. One major reason is the fact that the mediums for deploying CPS lack control and are used differently among stakeholders and user groups, making it difficult to define system boundaries. In other words, a system boundary of a CPS can be considered dynamic and unpredictable since, among other reasons, (1) users may access distributed systems differently depending on their demand, (2) elements of smart products evolve and advance (e.g. Apple's AppStore) and (3) the supply chains of smart products are affected by uncertainties similar to any other consumer product. Evidence of these trends can clearly be seen throughout the results of this paper as user groups behave differently depending on consumer attributes, e.g. data plans.

Furthermore, this study uses a bottom-up approach with empirical data from users and assessing the energy footprint of a particular technology. In order to extend this approach to a network level assessment, more transparency from the technologies themselves, e.g. power consumption of network infrastructure, must be provided. Till now, we only have estimates across the entire network over a given period of time, as in $[34,35]$. Breaking these grandiose numbers into per byte transmitted estimates would provide a means of understanding impact per user. This 
will allow the use of a dynamic life cycle assessment (LCA), in which user populations can be simulated to be representative of an actual smart technology deployed in a real-world scenario. In other smart products, e.g. tablets, video game consoles, an appropriate framework for procuring such data is clearly needed.

Additionally, in order to reach meaningful conclusions with regards to environmental foot-printing of ubiquitous technologies, rare earth metals, end-of-life implications human health risks, and other ecological risk categories must be closely investigated. This paper does not attempt to extend the analysis to encapsulate these issues, as this study only focuses on energy consumption. Other studies have tackled these issues from an industry-wide point of view [38-41], but have lacked the granularity and precision necessary to understand one specific deployed smart device. Also, in the past LCA practitioners may have used economic input-output life cycle analysis (EIO-LCA) in order to use industry-wide assessment to draw conclusions about a particular product [42]. The basis of EIO-LCA lies within the economic correlations between industries, and subindustries. This makes it particularly useful when exact lifecycle inventories are near impossible to collect in a timely fashion. Unfortunately, the results gathered from EIO-LCA have significant uncertainty in scaling down to a smartphone, for example.

\section{CONCLUSIONS}

This paper used empirical data of a large cohort of smartphone users to make user-group specific observations regarding behavior. Using an existing energy model of smartphone data access, the role of behavior in energy consumption was particularly investigated. New business scenarios were presented that do not curb user data usage but, at the same time, alleviate a significant fraction of the total energy consumption (i.e. 0.86-2.39 GWh yearly), and provide economic leverage for service providers. Insight into how this work could be applied to the emerging field of cyber-physical systems was also discussed.

\section{ACKNOWLEDGMENT}

The authors would like to thank Mobidia Technologies Inc. for providing the raw data that made this work possible. We are also grateful to Senthil Chandrasegaran for his helpful comments. The authors gratefully acknowledge the NSF IGERT on Sustainable Electronics (DGE 1144843) as well as another NSF Award (MANU 100619) for support of this research. The contents of this manuscript do not necessarily reflect the views and opinions of the NSF.

\section{REFERENCES}

[1] OECD/International Telecommunications Union, 2011. Mgovernment: Mobile technologies for responsive govern- ments and connected societies.

[2] Balasubramanian, N., Balasubramanian, A., and Venkataramani, A., 2009. "Energy consumption in mobile phones: a measurement study and implications for network applications". In Proceedings of the 9th ACM SIGCOMM conference on Internet measurement conference, ACM, pp. 280-293.

[3] PCmag, 2013. Pc magazine encyclopedia. URL: http://www.pcmag.com/encyclopedia/.

[4] Elmer-DeWitt, P., 2012. Nielsen: $66 \%$ percent of americans ages 24-35 own a smartphone. URL: http://tech.fortune.cnn.com/2012/02/20/nielsen-66-ofamericans-ages-24-35-own-a-smartphone.

[5] Lanzisera, S., Nordman, B., and Brown, R., 2012. "Data network equipment energy use and savings potential in buildings". Energy Efficiency, 5(2), pp. 149-162.

[6] Chan, C., Gygax, A., Wong, E., Leckie, C., Nirmalathas, A., and Kilper, D., 2012. "Methodologies for assessing the use-phase power consumption and greenhouse gas emissions of telecommunications network services". Environmental Science \& Technology.

[7] Xu, Q., Erman, J., Gerber, A., Mao, Z., Pang, J., and Venkataraman, S., 2011. "Identifying diverse usage behaviors of smartphone apps". In Proceedings of the 2011 ACM SIGCOMM conference on Internet measurement conference, ACM, pp. 329-344.

[8] Watson, R., Boudreau, M., and Chen, A., 2010. "Information systems and environmentally sustainable development: energy informatics and new directions for the is community". MIS quarterly, 34(1), p. 23.

[9] Mobidia, 2013. URL: http://www.mobidia.com/.

[10] Wehmeier, T., 2012. Understanding today's smartphone user: Demystifying data usage trends on cellular \& wi-fi networks.

[11] HBR, 2013. "Vision statement: How people really use mobile". Harvard Business Review: The Magazine, 91(1), pp. 30-31.

[12] Verkasalo, H., 2010. "Analysis of smartphone user behavior". In Mobile Business and 2010 Ninth Global Mobility Roundtable (ICMB-GMR), 2010 Ninth International Conference on, IEEE, pp. 258-263.

[13] Kivi, A., 2007. "Measuring mobile user behavior and service usage: methods, measurement points, and future outlook". Proceedings of the 6th Global Mobility Roundtable, pp. 1-2.

[14] Oliver, E., 2010. "The challenges in large-scale smartphone user studies". In Proceedings of the 2nd ACM International Workshop on Hot Topics in Planet-scale Measurement, ACM, p. 5.

[15] Falaki, H., Mahajan, R., Kandula, S., Lymberopoulos, D., Govindan, R., and Estrin, D., 2010. "Diversity in smartphone usage". In Proceedings of the 8th international 
conference on Mobile systems, applications, and services, ACM, pp. 179-194.

[16] Carroll, A., and Heiser, G., 2010. "An analysis of power consumption in a smartphone". In Proceedings of the 2010 USENIX conference on USENIX annual technical conference, USENIX Association, pp. 21-21.

[17] Tromp, N., Hekkert, P., and Verbeek, P.-P., 2011. "Design for socially responsible behavior: a classification of influence based on intended user experience". Design Issues, 27(3), pp. 3-19.

[18] Agichtein, E., Brill, E., Dumais, S., and Ragno, R., 2006. "Learning user interaction models for predicting web search result preferences". In Proceedings of the 29th annual international ACM SIGIR conference on Research and development in information retrieval, ACM, pp. 3-10.

[19] Campbell, J., Kleeman, D., and Ma, W., 2007. "The good and not so good of enforcing password composition rules". Information Systems Security, 16(1), pp. 2-8.

[20] Shiraishi, M., Washio, Y., Takayama, C., Lehdonvirta, V., Kimura, H., and Nakajima, T., 2009. "Using individual, social and economic persuasion techniques to reduce co 2 emissions in a family setting". In Proceedings of the 4th International Conference on Persuasive Technology, ACM, p. 13.

[21] Pérez, C., Cuadrado, M., and Cervera, A., 2009. "Understanding university library users' mistreatment of books". The Journal of Academic Librarianship, 35(2), pp. 177183.

[22] Wildervanck, C., 2000. "Driving the message home". In Transportation, Traffic Safety, and Health: Prevention and health: Third International Conference, Washington, USA, 1997, Vol. 3, Springer Verlag, p. 51.

[23] Watson, B., and Freeman, J., 2007. "Perceptions and experiences of random breath testing in queensland and the self-reported deterrent impact on drunk driving". Traffic Injury Prevention, 8(1), pp. 11-19.

[24] Radio, N., Zhang, Y., Tatipamula, M., and Madisetti, V., 2012. "Next-generation applications on cellular networks: trends, challenges, and solutions". Proceedings of the IEEE, 100(4), pp. 841-854.

[25] Heinemann, A., Kangasharju, J., and Muhlhauser, M., 2008. "Opportunistic data dissemination using real-world user mobility traces". In Advanced Information Networking and Applications-Workshops, 2008. AINAW 2008. 22nd International Conference on, IEEE, pp. 1715-1720.

[26] Shye, A., Scholbrock, B., Memik, G., and Dinda, P., 2010. "Characterizing and modeling user activity on smartphones: summary". In ACM SIGMETRICS Performance Evaluation Review, Vol. 38, ACM, pp. 375-376.

[27] Verkasalo, H., 2007. "Empirical insights on the evolution of the finnish mobile market". In Telecommunication TechnoEconomics, 2007. CTTE 2007. 6th Conference on, IEEE, pp. 1-9.

[28] Bowman, A., and Azzalini, A., 1997. "Applied smoothing techniques for data analysis".

[29] MATLAB, 2010. version 4.16 (R2011b). The MathWorks Inc., Natick, Massachusetts.

[30] Scharnhorst, W., Hilty, L., and Jolliet, O., 2006. "Life cycle assessment of second generation $(2 \mathrm{~g})$ and third generation $(3 \mathrm{~g})$ mobile phone networks". Environment international, 32(5), pp. 656-675.

[31] Meeker, M., and Wu, L., 2012. "Internet Trends. Presented at the D10 Conference by Kleiner Perkins Caufield and Byers".

[32] Duke Energy, 2006. Rate RS-Schedule for Residential and Farm Electric Service URL: http://www.dukeenergy.com/pdfs/DE-IN-Rate-RS.pdf.

[33] Lowensohn, J., 2012. iphone 5 estimated to cost 41 cents per year to charge.

[34] Koutitas, G., and Demestichas, P., 2009. "A review of energy efficiency in telecommunication networks". In Proc. Inter. Telecommunication Forum (TELFOR), pp. 2-7.

[35] Etoh, M., Ohya, T., and Nakayama, Y., 2008. "Energy consumption issues on mobile network systems". In Applications and the Internet, 2008. SAINT 2008. International Symposium on, IEEE, pp. 365-368.

[36] BBC News, 2013. Data Slowdown looms for smartphone users URL: http://www.bbc.co.uk/news/technology21118906.

[37] Stelter, B., 2012. Cable Operators to Expand Their Plan to Share Wi-Fi Hot Spots URL: http://mediadecoder.blogs.nytimes.com/2012/05/21/cableoperators-to-expand-their-plan-to-share-wi-fi-hot-spots.

[38] Köhler, A., Hilty, L., and Bakker, C., 2011. "Prospective impacts of electronic textiles on recycling and disposal". Journal of Industrial Ecology, 15(4), pp. 496-511.

[39] Bottner, H., Schischke, K., and Nissen, N., 2011. "Carbon footprinting of information technology products based on iso standards". In Consumer Electronics-Berlin (ICCEBerlin), 2011 IEEE International Conference on, IEEE, pp. 291-295.

[40] Fehske, A., Fettweis, G., Malmodin, J., and Biczok, G., 2011. "The global footprint of mobile communications: The ecological and economic perspective". Communications Magazine, IEEE, 49(8), pp. 55-62.

[41] Tseng, K., 2010. "Implementing and planning ict strategy for sustainable development in electrical electronics goods manufacture". In Manufacturing Automation (ICMA), 2010 International Conference on, IEEE, pp. 30-36.

[42] Matthews, H., and Small, M., 2000. "Extending the boundaries of life-cycle assessment through environmental economic input-output models". Journal of Industrial Ecology, 4(3), pp. 7-10. 